\title{
Gradenigo syndrome: A rare complication of otitis media
}

Paul Ferguson, M.D. ${ }^{1}$

\section{Author Affiliations:}

1. Department of Neurology, Marshall University Joan C. Edwards School of Medicine, Huntington, West Virginia

The authors have no financial disclosures to declare and no conflicts of interest to report.

\section{Corresponding Author:}

Paul Ferguson, MD

Interim Chairman, Department of Neurology

Marshall University Joan C. Edwards School of Medicine

Huntington, West Virginia

Email: paul.ferguson@marshall.edu 


\section{Abstract:}

Gradenigo syndrome (petrous apicitis or petrositis) is a rare complication of otitis media that if left unrecognized and subsequently untreated will result in severe neurologic sequelae. Gradenigo syndrome is clinically represented by the triad of otitis media, ipsilateral abducens nerve palsy and dysasthesia within the V1 and V2 divisions of the trigeminal nerve branches. We describe a 4 year-old patient who presented with headache, facial pain, diplopia and a recent history of upper respiratory tract symptomatology. Examination revealed an abducens palsy with subsequent magnetic resonance imaging demonstrating inflammation in the left petrous apex and along the tracts of the 6th, 7th, and 8th cranial nerves. The patient was treated with intravenous antibiotics along with myringotomy and had complete resolution of his symptoms.

\section{Key words:}

Gradenigo syndrome, petrous apicitis, petrositis, otitis media, multiple cranial nerve palsies

\section{Introduction:}

Gradenigo syndrome was first described by Guiseppe Gradenigo in 1907. The condition is also referred to as petrous apicitis or petrositis in some texts. It is a clinical triad of otitis media symptomatology, ipsilateral abducens nerve palsy and pain in the regions of the first and second divisions of the trigeminal nerve. ${ }^{1}$ It is a complication of otitis media that occurs due to the local spread of infection to the petrous apex of the temporal bone. The proximity of the 5th and 6th nerve ganglions to the petrous apex results in the above mentioned symptoms. After the advent of antibiotics the incidence of this syndrome has decreased markedly and is estimated to be 2 per 100,000 children with otitis media. ${ }^{2,3}$

We present a rare case of Gradenigo syndrome along with the pertinent literature review.

\section{Case Report:}

A 4 year-old male referred to the neurology clinic for same-day assessment from his primary care physician with headaches, facial pain, diplopia, and left periorbital swelling. Approximately four weeks earlier the patient was treated for an episode of otitis media with Augmentin $^{\mathrm{TM}}$. After completing the prescribed antibiotic course he began having headaches in the left temporal and frontal area. The patient's headaches became progressively more frequent. Five days prior to being seen in the neurology clinic he was evaluated in the emergency department for left periorbital swelling and episodic diplopia. He was then initiated on Bactrim ${ }^{\mathrm{TM}}$ for presumed sinusitis. On the day of initial neurology evaluation the patient continued to complain of headaches, facial pain, and diplopia. Physical exam was significant for left abducens nerve palsy along with left periorbital swelling and erythema. No other neurological deficits were noted. In view of the neurological findings an urgent magnetic resonance imaging (MRI) with and without gadolinium was ordered to evaluate for an intracranial abscess or venous sinus thrombosis. It revealed inflammatory changes in the left petrous apex and the mastoid extending in to the cerebellopontine angle. Inflammatory changes were also noted in the left 6th, 7th and 8th nerve complexes (Fig 1). Magnetic resonance venogram (MRV) of the head failed to demonstrate venous sinus thrombosis. CT of the inner ear showed inflammatory erosion of the petrous bone and enhancement of the 6th cranial nerve consistent with the findings noted on the MRI. The patient was then diagnosed with Gradenigo syndrome and admitted to the pediatric inpatient service. Pediatric infectious disease was consulted and he was started on vancomycin, ceftriaxone and metronidazole. He 
was also started on decadron to reduce the swelling. He was evaluated by ENT and bilateral myringotomy tubes were placed. The patient's periorbital swelling and lateral gaze palsy improved significantly. He was sent home to complete a 4-week course on linezolid, metronidazole and ceftriaxone. Upon follow up the patient's symptoms completely resolved.

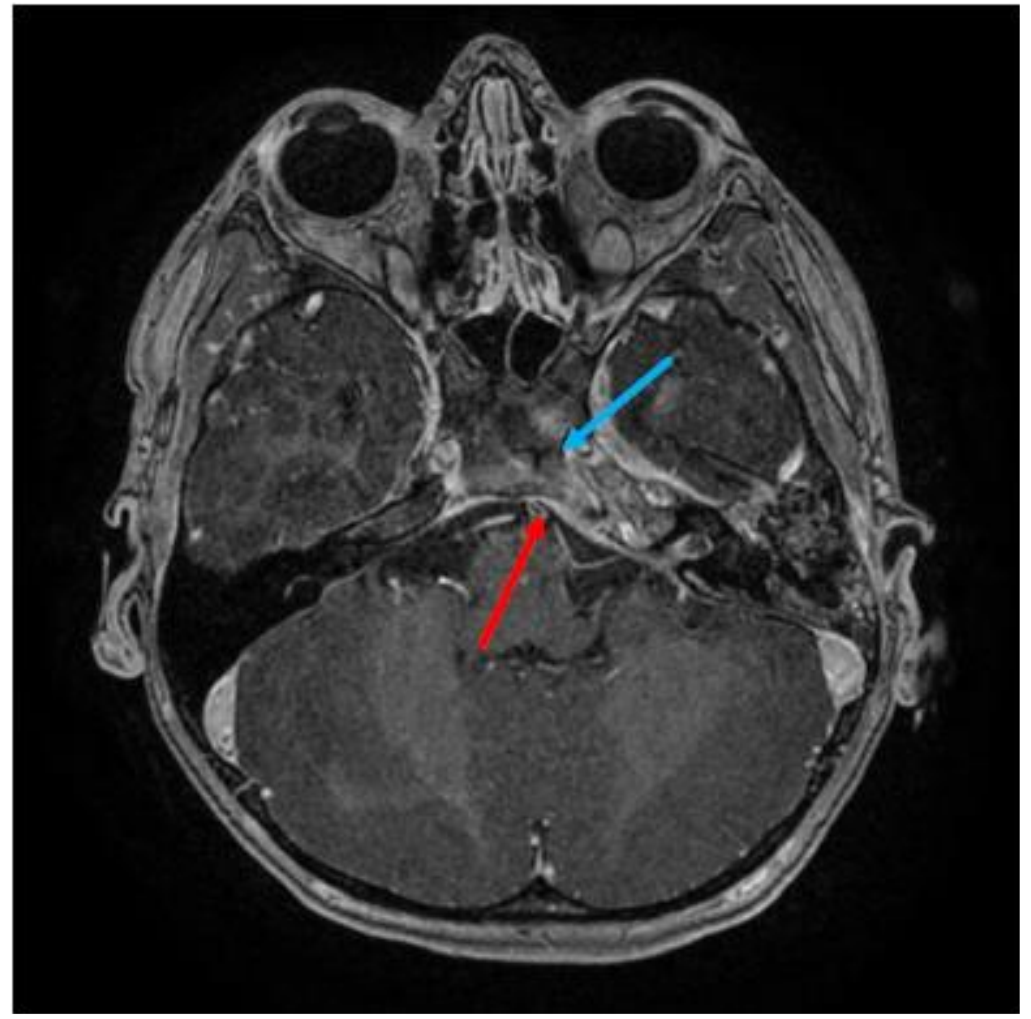

Figure 1: MRI of the brain showing enhancement of the left pterosal apex (red arrow). Enhancement is also noted along the tract of the trigeminal nerve within Meckel's Cave (blue arrow).

\section{Discussion:}

Gradenigo syndrome is due to the inflammation of the petrous apex of the temporal bone and is a rare complication of otitis media. The petrous apex, unlike the mastoid cells, is only pneumatized in approximately $33 \%$ of patients. This absence of pneumatization is suspected to play a role in the spread of infection. Additionally infection could also spread by direct extension or by hematological dissemination through the venous plexuses. ${ }^{2}$

The anatomical location of the petrous apex is such that infection in this region can cause serious complications including meningitis, empyema, brain abscess and venous sinus thrombosis; therefore, one must be vigilant for these rare and serious complications. Petrous apicitis infections are often difficult to diagnose clinically given that symptoms often coincide with many upper respiratory tract symptoms including facial pain, ear fullness and headache. $^{2}$ The time lag between otological symptoms and neurological symptoms may vary from 1 week to 2-3 months; in our case the time lag was approximately 1 month. ${ }^{3}$

Although a contrasted head CT has high sensitivity for detection of inflammatory changes within the petrous apex, enhanced MRI is superior for detecting meningeal enhancement and other intracranial complications described above. A magnetic resonance venogram may also be performed to rule out comorbid venous sinus thrombosis which may occur as a complication of this entity. ${ }^{2,4,5,6}$ 
The management of Gradenigo syndrome includes medication, surgical, and conservative approaches described in the literature..$^{3,7,8,9,10}$ The combination of aggressive intravenous antibiotics therapy with myringotomy was employed with success in the above described case with realization of complete symptom resolution within four weeks.

\section{Conclusion:}

Although Gradenigo syndrome is rare, physicians must be aware of the symptom complex as to prevent delay in diagnostic evaluation as any postponement in diagnosis can lead to potentially lethal complications. The diagnosis of this syndrome can pose difficulties as the components of the triad may not occur at the same time and therefore a careful history and physical exam are of utmost importance. 


\section{References:}

1. Gradenigo G: Uber die paralyse des n. Abduzens bei otitis. Arch F Ohrenheilk. 1907;74:149-158.

2. Jacobsen CL, Bruhn MA, Yavarian Y, Gaihede ML. Mastoiditis and Gradenigo's Syndrome with anaerobic bacteria. BMC Ear Nose Throat Disord. 2012;14:12:10.

3. Gradenigo syndrome and petrositis in a child. Favier M, Bessou P, Franco-Vidal V, Pédespan JM. Arch Pediatr. 2015 Mar;22(3):283-6.

4. Marianowski R, Rocton S, Ait-Amer JL, Morisseau-Durand MP, Manach Y. Conservative management of Gradenigo syndrome in a child. Int J Pediatr Otorhinolaryngol. 2001;57(1):79-83.

5. Jackler RK, Parker DA. Radiographic differential diagnosis of petrous apex lesions. Am J Otol. 1992;13(6):561-74.

6. Kantas I, Papadopoulou A, Balatsouras DG, Aspris A, Marangos N. Therapeutic approach to Gradenigo's syndrome: a case report. J Med Case Rep. 2010;24:4:151.

7. Minotti AM, Kountakis SE. Management of abducens palsy in patients with petrositis. Ann Otol Rhinol Laryngol. 1999; 108(9):897-902.

8. Kong SK, Lee IW, Goh EK, Park SE. Acute otitis media-induced petrous apicitis presenting as the Gradenigo syndrome: successfully treated by ventilation tube insertion. Am J Otolaryngol. 2011;32(5):445-7.

9. Mystery Case: Cholesterol granuloma of the petrous apex in Gradenigo syndrome. Lattanzi S, Cagnetti C, Di Bella P, Provinciali L.Neurology. 2015 Apr 28;84(17):e122-3.

10. Favier M, Bessou P, Franco-Vidal V, Pédespan JM. Gradenigo syndrome and petrositis in a child. Arch Pediatr. 2015 Mar;22(3):283-6. 